\title{
Increased expression of monoamine oxidase $A$ is associated with epithelial to mesenchymal transition and clinicopathological features in non-small cell lung cancer
}

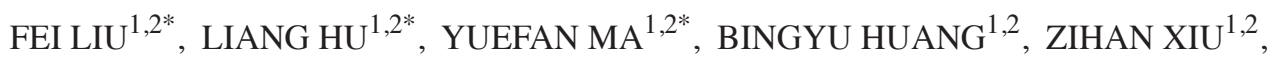 \\ PEIHUA ZHANG ${ }^{3}$, KEYUAN ZHOU ${ }^{1,2}$ and XUDONG TANG ${ }^{1,2}$ \\ ${ }^{1}$ Institute of Biochemistry and Molecular Biology, Guangdong Medical University, Zhanjiang, \\ Guangdong 524023; ${ }^{2}$ Guangdong Provincial Key Laboratory of Medical Molecular Diagnostics, \\ Guangdong Medical University, Dongguan, Guangdong 523808; ${ }^{3}$ Institute of Plastic Surgery, \\ Affiliated Hospital of Guangdong Medical University, Zhanjiang, Guangdong 524001, P.R. China
}

Received April 28, 2017; Accepted December 14, 2017

DOI: $10.3892 / \mathrm{ol} .2017 .7683$

\begin{abstract}
Monoamine oxidase A (MAOA), a mitochondrial enzyme, is closely associated with neurological disorders. Recently, MAOA has been linked to the progression of prostate cancer, hepatocellular carcinoma, and cholangiocarcinoma. However, MAOA was reported to have different effects on the progression of these types of cancer, and the role of MAOA in non-small cell lung cancer (NSCLC) progression remains unclear. The present study determined the expression of MAOA and epithelial to mesenchymal transition (EMT) markers in 45 pairs of NSCLC and matched non-tumor adjacent lung tissues, and further analyzed the correlation between MAOA expression and the EMT or the development of clinicopathological features. The results demonstrated that protein and mRNA expression levels of MAOA in NSCLC tissues were higher than those observed in the matched non-tumor adjacent lung tissues. Furthermore, the increased MAOA expression in NSCLC tissues was positively correlated with $\mathrm{N}$-cadherin $(r=0.525$, $\mathrm{P}=0.002)$, Slug $(r=0.515, \mathrm{P}=0.001)$, and Twist $(r=0.448$, $\mathrm{P}=0.008$ ) expressions, but negatively correlated with E-cadherin expression $(r=-0.387, \mathrm{P}=0.01)$. Additionally, the elevated MAOA expression in NSCLC tissues was associated with late stage NSCLC $(Z=-2.596, P=0.029)$ and
\end{abstract}

Correspondence to: Professor Xudong Tang, Institute of Biochemistry and Molecular Biology, Guangdong Medical University, 2 Wenming Donglu, Xiashan, Zhanjiang, Guangdong 524023, P.R. China

E-mail: tangxudong2599@126.com; txd@gdmu.edu.cn

${ }^{*}$ Contributed equally

Key words: monoamine oxidase A, non-small cell lung cancer, epithelial to mesenchymal transition, clinicopathological feature, clinical stage, lymph node metastasis lymph node metastases $(\mathrm{Z}=-2.378, \mathrm{P}=0.020)$. These findings suggest that MAOA may have a role in promoting NSCLC progression by mediating EMT.

\section{Introduction}

Lung cancer, the leading cause of cancer-related deaths worldwide, is commonly divided into two categories, small cell lung cancer (SCLC) and non-SCLC (NSCLC), depending on its degree of differentiation and morphological characteristics $(1,2)$. NSCLC accounts for $\sim 80 \%$ of primary lung cancers, including squamous cell carcinoma, adenocarcinoma, and large cell carcinoma (3). The 5-year survival rate of NSCLC is only $7 \%$ (4). Moreover, lymph nodes and distant organ metastasis are the main reasons leading to treatment failure in NSCLC patients with radical resection $(5,6)$.

Epithelial to mesenchymal transition (EMT), a reversible biological process, is characterized by the loss of epithelial cell junction proteins (E-cadherin, Zo-1) $(7,8)$, the gain of mesenchymal markers (vimentin, N-cadherin) $(9,10)$, and the activation of transcription factors (Snail1, Slug, ZEB1, Twist) (11-15). Accumulating evidence indicates that EMT enhances tumor invasion, distant metastasis, and chemotherapy resistance in NSCLC, underscoring the need for a comprehensive understanding of the EMT function in NSCLC progression (16-19).

Monoamine oxidase A (MAOA), a mitochondria-bound enzyme, catalyzes the oxidative deamination of dietary amines and monoamine neurotransmitters, such as serotonin, norepinephrine, and dopamine $(20,21)$. The functions of MAOA have been extensively studied in the context of neurological disorders, including mental depression, aggressive behaviors, and Parkinson's disease $(22,23)$. Recent studies have indicated the role of MAOA in the progression of prostate cancer (24-30), hepatocellular carcinoma (HCC)(31), and cholangiocarcinoma (32). High Gleason grade or poorly differentiated prostate cancer exhibited increased MAOA expression (24), and the increased MAOA promoted prostate cancer metastasis $(25,26)$. Furthermore, 
overexpression of MAOA was found to dramatically downregulate the expression of E-cadherin and upregulate the expression of vimentin and Twist at both mRNA and protein levels in prostate cancer (25). These studies suggested that MAOA might promote the progression of prostate cancer by mediating EMT. However, conflicting results were reported for HCC (31) and cholangiocarcinoma (32). Therefore, the role of MAOA may vary across cancer types, and therefore, it is essential to further understand the function of MAOA in other cancers.

Little is known about the function of MAOA in NSCLC. Accordingly, in this study, we investigated the expression of MAOA in NSCLC tissues and analyzed the association between the expression of MAOA and EMT or the development of clinicopathological features. We found for the first time, to the best of our knowledge, that MAOA protein and mRNA expressions in NSCLC tissues were significantly higher than those observed in the matched non-tumor adjacent lung tissues, and the increased MAOA expression was related to EMT, clinical stages, and lymph node metastases in NSCLC, suggesting that MAOA may be involved in mediating the progression of NSCLC.

\section{Materials and methods}

Reagents. Rabbit anti-human MAOA monoclonal antibody was obtained from Abcam (ab126751; Cambridge, UK). Mouse anti-human E-cadherin monoclonal antibody, rabbit anti-human vimentin, N-cadherin, Snaill, Slug, Zo-1, ZEB1 and Twist monoclonal antibodies, and horseradish peroxidase (HRP)-conjugated secondary antibodies were purchased from Cell Signaling Technology, Inc. (Beverly, MA, USA). The RNA extraction kit (RNAprep Pure FFPE kit) was purchased from Tiangen Biotech Co., Ltd. (Beijing, China). The reverse transcription (RT) kit (PrimeScript ${ }^{\mathrm{TM}}$ RT reagent kit) and qPCR analysis kit (SYBR Premix Ex $\mathrm{Taq}^{\mathrm{TM}}$ II) were obtained from Takara Biotechnology Co., Ltd. (Dalian, China).

NSCLC patients and control cases. NSCLC tissue specimens were obtained from 45 patients who were definitively diagnosed with NSCLC and had undergone curative surgery between 2007 and 2010 at the Affiliated Hospital of Guangdong Medical University (Guangdong, China). The matched non-tumor adjacent lung tissues $(1 \mathrm{~cm}$ from the tumor) were also collected from the same patients, as the controls. Among these patients, the complete clinicopathological and histopathological data were collected from 30 cases. The patients that met the following criteria were enrolled. First, the patients were definitively diagnosed with NSCLC based on histological examinations. Second, the patients had not received chemotherapy, immunotherapy, or radiotherapy before pulmonary lobectomy. Third, the patients showed normal hepatic and renal functions and no abnormality of the endocrine system.

Ethics approval. Either the patients or their close relatives provided informed written consents. Our investigation received the ethic approval from the local Committee of the Affiliated Hospital of Guangdong Medical University. All clinical investigations were performed according to the principles defined by the Declaration of Helsinki.

Immunohistochemistry. Immunohistochemical staining was performed on paraffin-embedded tissue specimens, including NSCLC and matched non-tumor adjacent lung tissues, from 45 cases. Briefly, paraffin-embedded tissue specimens were cut into $4-\mu \mathrm{m}$ sections, transferred onto Superfrost Ultra Plus slides, and placed in a $60^{\circ} \mathrm{C}$ oven overnight. The slides were deparaffinized with xylene, rehydrated in a descending alcohol series $(100,95,90,80$ and $70 \%$ ), and then rinsed with sterile distilled water for $5 \mathrm{~min}$. Antigen retrieval was performed by boiling the tissue sections in citrate buffer $(10 \mathrm{mM}$ trisodium citrate, $0.05 \%$ Tween-20, $\mathrm{pH}=6$ ) for $10 \mathrm{~min}$. The slides were further treated with $3 \%$ hydrogen peroxide for $15 \mathrm{~min}$ to inactivate any endogenous peroxidase activity. After rinsing in phosphatebuffered saline (PBS), non-specific sites were blocked with normal goat serum for $15 \mathrm{~min}$. The slides were subsequently incubated with primary antibodies (1:100) overnight at $4^{\circ} \mathrm{C}$. One slide was incubated with PBS, as the negative control. After washing with precooled PBS, the slides were incubated with a secondary biotinylated antibody for $15 \mathrm{~min}$ at room temperature. The slides were washed with precooled PBS three times, and then treated with a streptavidin-biotin complex for $15 \mathrm{~min}$ at room temperature. The slides were then stained with diaminobenzidine (DAB), and counterstained with hematoxylin, before being observed and analyzed in a double-blind manner, under light microscopy, by two senior pathologists. Ten randomly selected fields were examined at x400 magnification and 100 cancer cells were counted in each field (total 10,000 cells) to determine the proportion of positive cells. A semi-quantitative analysis was performed to evaluate the protein expression levels as described previously $(33,34)$. In brief, staining intensity was scored on a scale of 0 to 3, 0 for no intensity, 1 for low intensity (light yellow), 2 for moderate intensity (claybank), and 3 for high intensity (sepia). The cell positivity was scored on a scale of 0 to 4 : $0,<5 \%$ cells stain-positive; 1,5 to $25 \%$ cells stain-positive; 2, 26 to $50 \%$ cells stain-positive; 3,51 to $75 \%$ cells stain-positive; and $4,>75 \%$ cells stain-positive. The scores obtained relative staining intensity and proportion of positive cells were multiplied together to generate a final score ranging from 0 to 12 , interpreted as follows: 0 , negative (-); 1 to 4 , weakly positive (+); 5 to 8 , moderately positive $(++) ; 9$ to 12 , strongly positive $(+++)$. The scores were evaluated by two pathologists.

$R T-q P C R$. Total RNA was extracted from paraffin-embedded tissue specimens using the TIANGEN RNAprep Pure FFPE kit and then converted to cDNA using the PrimeScript ${ }^{\mathrm{TM}}$ RT reagent kit (both from Tiangen Biotech Co., Ltd.). qPCR analysis was performed using SYBR Premix Ex Taq ${ }^{\mathrm{TM}}$ II (Takara Biotechnology Co., Ltd.), according to the manufacturer's instructions. All the primers were synthesized by Takara Biotechnology Co., Ltd., and are listed in Table I. The housekeeping gene $\beta$-actin was used as an internal control to normalize mRNA levels. The optimum reaction conditions for qPCR were as follows: Pre-treatment at $42^{\circ} \mathrm{C}$ for $5 \mathrm{~min}$, initial denaturation at $95^{\circ} \mathrm{C}$ for $10 \mathrm{sec}$, followed by 40 cycles at $95^{\circ} \mathrm{C}$ 
Table I. Primers for real-time quantitative PCR.

\begin{tabular}{|c|c|c|c|}
\hline GenBank no. & Genes & Primer sequence $\left(5^{\prime}-3^{\prime}\right)$ & Length (bp) \\
\hline NM_000240.3 & $M A O A$ & $\begin{array}{l}\text { Forward primer AGTGAGCGAACGGATAATGG } \\
\text { Reverse primer TGTTCATGGTTCAGCGTCTC }\end{array}$ & 114 \\
\hline NM_004360.4 & E-cadherin & $\begin{array}{l}\text { Forward primer TTGCTACTGGAACAGGGACAC } \\
\text { Reverse primer CCCGTGTGTTAGTTCTGCTGT }\end{array}$ & 179 \\
\hline NM_003257.4 & Zo-1 & $\begin{array}{l}\text { Forward primer GGATGTTTATCGTCGCATTGTA } \\
\text { Reverse primer AAGAGCCCAGTTTTCCATTGTA }\end{array}$ & 158 \\
\hline NM_001792.4 & $N$-cadherin & $\begin{array}{l}\text { Forward primer TTATCCTTGTGCTGATGTTTGTG } \\
\text { Reverse primer TCTТCTTCТССТССАССТTСТTC }\end{array}$ & 139 \\
\hline NM_003380.3 & Vimentin & $\begin{array}{l}\text { Forward primer TGGCACGTCTTGACCTTGAA } \\
\text { Reverse primer GGTCATCGTGATGCTGAGAA }\end{array}$ & 176 \\
\hline NM_005985.3 & Snail1 & $\begin{array}{l}\text { Forward primer TCCTTCGTCCTTCTCСТСТАСТT } \\
\text { Reverse primer TGTTGCAGTATTTGCAGTTGAAG }\end{array}$ & 155 \\
\hline NM_003068.4 & Slug & $\begin{array}{l}\text { Forward primer GCCTTTTTCTTGCCCTCAC } \\
\text { Reverse primer GGTTTTGGAGCAGTTTTTGC }\end{array}$ & 115 \\
\hline NM_030751.5 & ZEBI & $\begin{array}{l}\text { Forward primer TCCCCATCACCTCTAAACCTT } \\
\text { Reverse primer CCCTGTTGCTTTGGTAGTGAA }\end{array}$ & 122 \\
\hline NM_000474.3 & Twist & $\begin{array}{l}\text { Forward primer AGTCCGCAGTCATACGAGGAG } \\
\text { Reverse primer GACCTAGTAGAGGAAGTCGATG }\end{array}$ & 146 \\
\hline NM_001101.3 & $\beta$-actin & $\begin{array}{l}\text { Forward primer TGACGTGGACATCCGCAAAG } \\
\text { Reverse primer CTGGAAGGTGGACAGCAGGG }\end{array}$ & 186 \\
\hline
\end{tabular}

MAOA, monoamine oxidase A.

for $5 \mathrm{sec}$, and $60^{\circ} \mathrm{C}$ for $31 \mathrm{sec}$. The experiment was carried out in triplicate.

Statistical analysis. SPSS 19.0 Windows software was used for statistical analysis. Quantitative data were presented as the mean \pm SD. The categorical variables were presented as frequency and percent rates, and the positive rates from two groups were compared using Chi-square $\left(\chi^{2}\right)$ test or Fisher exact probabilities $(n<40$ or $T<1)$. Wilcoxon rank sum test was used to perform the statistical analysis on ordinal data. Spearman rank correlation coefficient was employed for the correlation analysis. P-value $<0.05$ was considered to indicate a statistically significant difference.

\section{Results}

Expressions of MAOA and EMT markers in NSCLC and matched non-tumor adjacent lung tissues. Previous studies have demonstrated that prostate cancer and HCC exhibit completely different MAOA expression levels (24,31), and to date, MAOA expression has not been reported in NSCLC. To investigate the expressions of MAOA and EMT markers in NSCLC tissues, immunohistochemical staining was performed in 45 pairs of NSCLC and patient-matched non-tumor adjacent lung tissues. The protein expressions of MAOA, N-cadherin, vimentin, Snail1, Slug, ZEB1, and Twist were obviously enhanced in NSCLC tissues (Fig. 1A). The positive expression rates of MAOA, N-cadherin, vimentin,
Snail1, Slug, ZEB1, and Twist in NSCLC tissues were higher than those observed in the matched non-tumor adjacent lung tissues $(\mathrm{P}<0.01$, Fig. 1B; Table II), while the positive expression rates of E-cadherin, Zo-1, and EMT epithelial makers determined in NSCLC tissues were lower than those observed in adjacent normal lung tissues (Fig. 1A and B; Table II). To further investigate the mRNA expressions of MAOA and EMT markers, NSCLC and matched adjacent normal lung tissues from 30 cases were selected for RT-qPCR. Our results showed that the mRNA levels of MAOA, N-cadherin, vimentin, Snail1, Slug, ZEB1, and Twist in NSCLC tissues were significantly higher than those detected in non-tumor adjacent tissues. As expected, a significant decrease in E-cadherin and Zo-1 mRNA expressions was observed in NSCLC tissues $(\mathrm{P}<0.01$; Fig. 1C).

Moreover, the statistical distribution results further demonstrated that MAOA, vimentin, and Snaill protein expressions were significantly stronger in NSCLC tissues than in adjacent normal lung tissues, whereas E-cadherin protein expression displayed the opposite trend $(\mathrm{P}<0.05$; Fig. 2).

Correlation between the expressions of MAOA and EMT markers in NSCLC. To study the role of MAOA expression in the EMT of NSCLC, a Spearman rank correlation coefficient test was performed to analyze the correlation between the expressions of MAOA and EMT markers in NSCLC. As described in Table III, although there was no relationship between the expression of MAOA and the expressions 
Table II. The positive rates of protein expression of MAOA and EMT markers in NSCLC and non-tumor adjacent lung tissues.

\begin{tabular}{|c|c|c|c|c|}
\hline Proteins & NSCLC tissues (\%) & $\begin{array}{l}\text { Non-tumor adjacent } \\
\text { lung tissues }(\%)\end{array}$ & $\chi^{2}$ & P-value \\
\hline MAOA & $71.1(32 / 45)$ & $40.0(18 / 45)$ & 8.802 & 0.003 \\
\hline E-cadherin & $46.7(21 / 45)$ & $77.8(35 / 45)$ & 9.265 & 0.002 \\
\hline Zo-1 & $51.1(23 / 45)$ & $91.1(41 / 45)$ & 17.524 & 0.0001 \\
\hline $\mathrm{N}$-cadherin & $68.9(31 / 45)$ & $26.7(9 / 45)$ & 21.780 & 0.0001 \\
\hline Vimentin & $44.4(20 / 45)$ & $17.8(8 / 45)$ & 7.465 & 0.006 \\
\hline Snail1 & $62.2(28 / 45)$ & $33.3(15 / 45)$ & 7.526 & 0.006 \\
\hline Slug & $55.6(25 / 45)$ & $20.0(9 / 45)$ & 12.101 & 0.001 \\
\hline ZEB1 & $53.3(24 / 45)$ & $15.6(7 / 45)$ & 14.221 & 0.0001 \\
\hline Twist & $64.4(29 / 45)$ & $22.2(10 / 45)$ & 16.335 & 0.0001 \\
\hline
\end{tabular}

MAOA, monoamine oxidase A; EMT, epithelial to mesenchymal transition; NSCLC, non-small cell lung cancer.

A

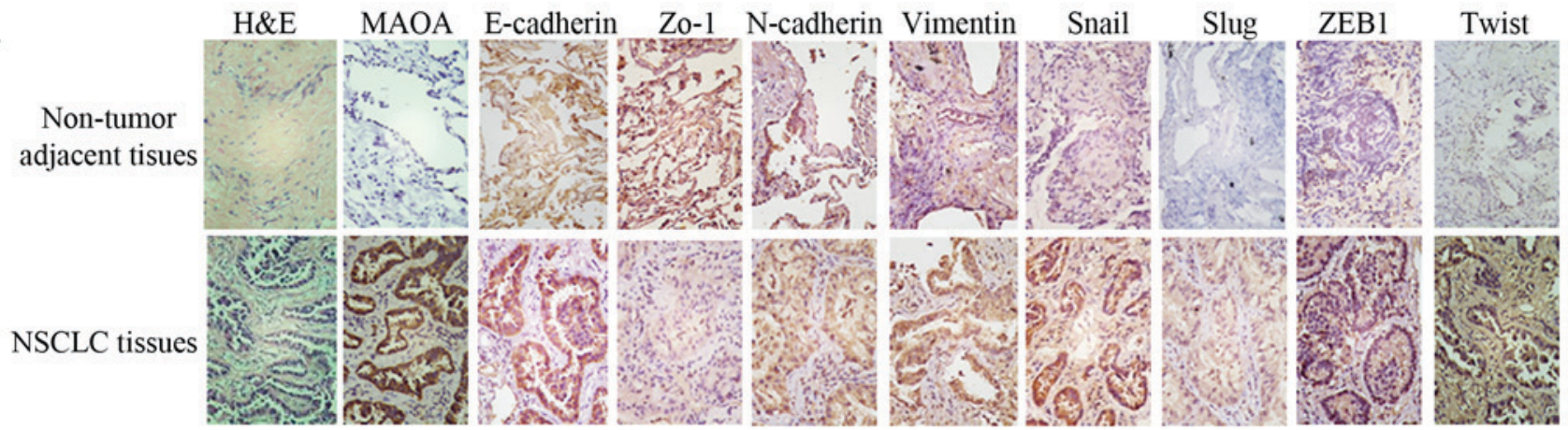

$\mathrm{B}$

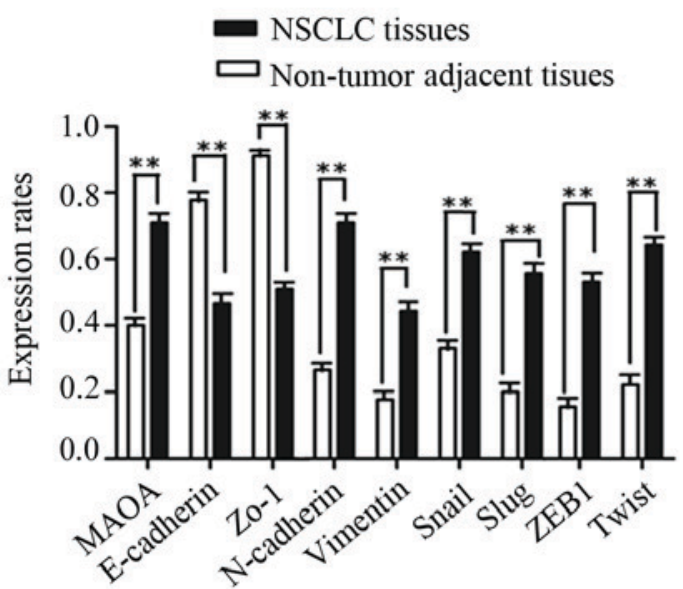

C
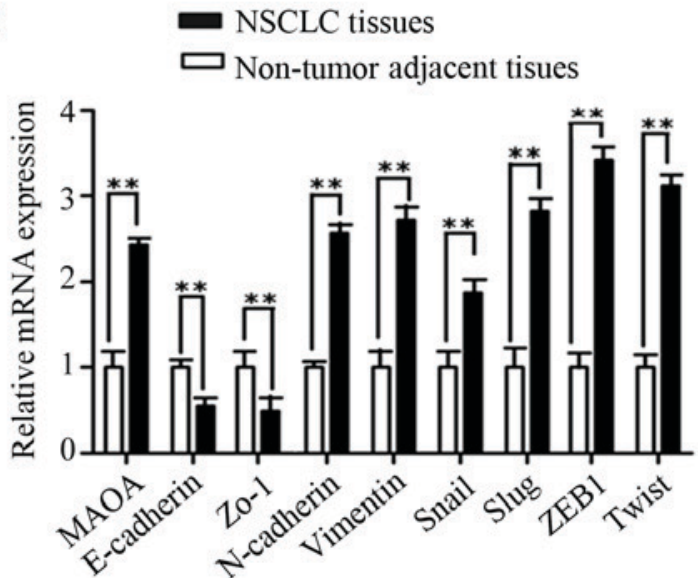

Figure 1. The expressions of MAOA and EMT markers in NSCLC and the matched non-tumor adjacent lung tissues. Immunohistochemistry and RT-qPCR were performed to analyze the expressions of MAOA and EMT markers. (A) The representative results of immunohistochemistry. Original magnification, $\mathrm{x} 400$. (B) The positive rates of protein expression, ${ }^{* *} \mathrm{P}<0.01$. (C) RT-qPCR results, ${ }^{* *} \mathrm{P}<0.01$. MAOA, monoamine oxidase A; EMT, epithelial to mesenchymal transition; NSCLC, non-small cell lung cancer.

of vimentin, Snail1, Zo-1, or ZEB1 (P>0.05; Table III), the expression of MAOA was positively correlated with the expressions of the EMT mesenchymal marker N-cadherin $(r=0.525, \mathrm{P}=0.002)$ and the EMT transcription factors Slug $(r=0.515, \mathrm{P}=0.001)$ and Twist $(r=0.448, \mathrm{P}=0.008)$. Accordingly, MAOA expression was negatively correlated with the expression of the epithelial maker E-cadherin ( $r=-0.387, \mathrm{P}=0.01$; Table III).

Correlation between MAOA expression and the development of clinicopathological features in NSCLC. Thirty of the patients who were definitively diagnosed with NSCLC, and 
A

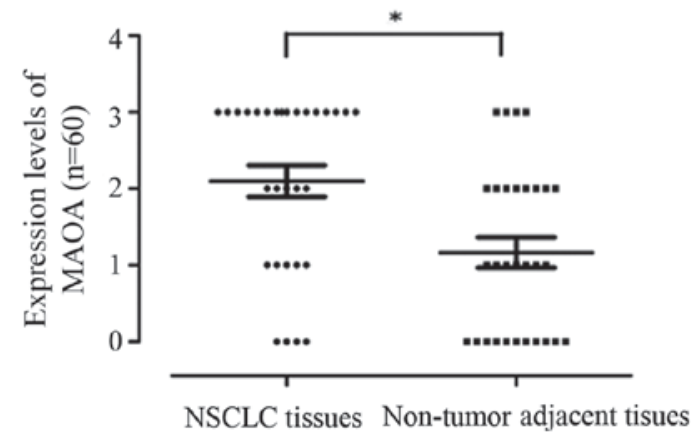

C

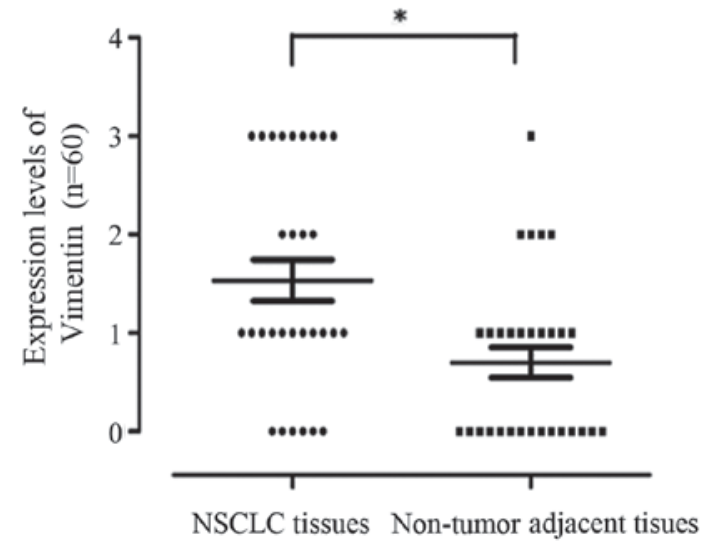

B

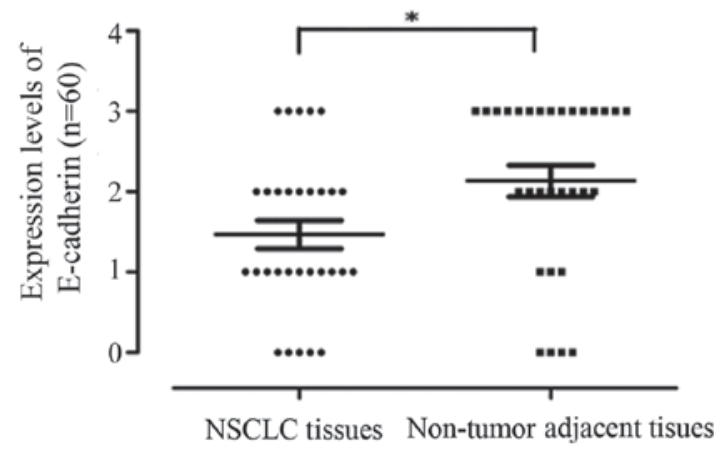

D

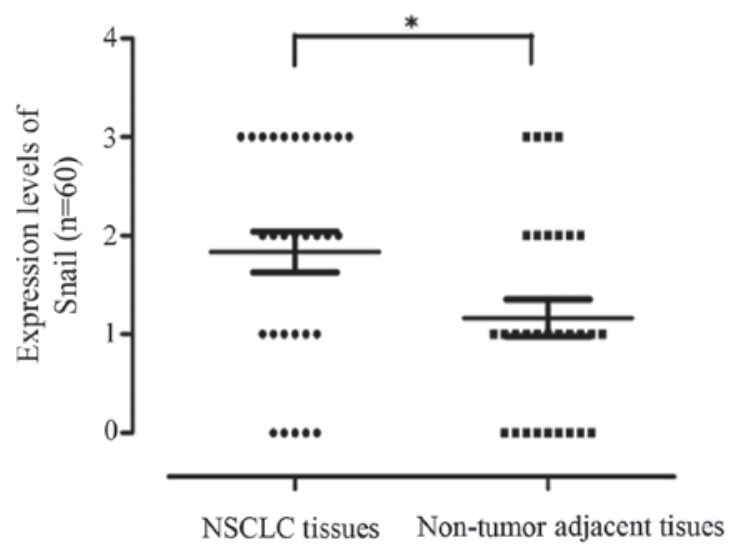

Figure 2. The statistical distribution of MAOA, E-cadherin, vimentin, and Snaill expression levels in NSCLC and the matched non-tumor adjacent lung tissues. Wilcoxon rank sum test was employed for analysis of statistical distribution of protein expression levels of MAOA, E-cadherin, vimentin, and Snail1. (A) MAOA, (B) E-cadherin, (C) vimentin, and (D) Snail1. * $\mathrm{P}<0.05$. MAOA, monoamine oxidase A; NSCLC, non-small cell lung cancer.

offered complete clinicopathological and histopathological data, were enrolled to analyze the correlation between MAOA expression and the clinicopathological features observed in NSCLC. A Wilcoxon rank sum test was employed to carry out the analysis of statistical distribution on the data obtained regarding the protein expression levels. Our results showed that the positive rate of MAOA expression in stage III was higher than that measured in stages I and II $(Z=-2.596$, $\mathrm{P}=0.029$; Table IV). Additionally, the lymph node metastasis group exhibited a stronger MAOA expression level than the controls with no metastasis $(\mathrm{Z}=-2.378, \mathrm{P}=0.020$; Table IV). These results indicated that MAOA expression was significantly correlated with clinical stages and lymph node metastases. However, MAOA expression was not influenced by sex, age, degree of differentiation, or histological types (P>0.05; Table IV).

\section{Discussion}

MAOA, a monoamine neurotransmitter degrading enzyme, is well-known to be closely associated with impulsive aggressively, anxiety, depression, among other emotions, and is considered as an indicator of psychological status $(22,23,35)$. Recently, several studies have been focusing on the relationship between MAOA expression and cancers (24-32). However, conflicting results were reported across different types of cancer, including prostate cancer (24-30), HCC (31), and cholangiocarcinoma (32). MAOA was demonstrated as being highly expressed in high-grade aggressive prostate cancer, and capable of mediating prostate tumorigenesis and metastasis (24-27). Recently, MAOA was reported as a novel decision maker in apoptosis and autophagy processes occurring within hormone refractory neuroendocrine prostate cancer cells (28). Moreover, clorgyline, a MAOA inhibitor, was found to exhibit anti-oncogenic and pro-differentiation effects on high-grade prostate cancer cells (29), and the MAOA inhibitor-near-infrared dye conjugate was reported to reduce prostate tumor growth (30). These findings suggest that MAOA might play a key role in mediating prostate cancer progression. However, Li et al demonstrated that MAOA expression was remarkably downregulated in clinical HCC tissue samples (31), and that MAOA suppressed HCC metastasis by inhibiting the adrenergic system and its transactivation of EGFR signaling (31). Huang et al also found that MAOA expression was inhibited by coordinated epigenetic and IL-6-driven events in human cholangiocarcinoma (32), and that overexpression of MAOA suppressed cholangiocarcinoma growth and invasion (32). In the present study, we demonstrated for the first time to our knowledge that MAOA protein and mRNA expression levels, positive rates, and statistical distribution in NSCLC tissues were dramatically higher than those recorded in the matched non-tumor 
Table III. The correlation between MAOA expression and EMT in NSCLC.

\begin{tabular}{|c|c|c|c|c|c|c|}
\hline \multirow[b]{2}{*}{ Proteins } & \multicolumn{2}{|c|}{ MAOA } & \multirow[b]{2}{*}{ Total } & \multirow[b]{2}{*}{$\chi^{2}$} & \multirow[b]{2}{*}{ P-value } & \multirow[b]{2}{*}{$\mathrm{r}$} \\
\hline & + & - & & & & \\
\hline E-cadherin & & & & 6.724 & 0.010 & -0.387 \\
\hline+ & 11 & 10 & 21 & & & \\
\hline - & 21 & 3 & 24 & & & \\
\hline Zo-1 & & & & 0.795 & 0.372 & -0.133 \\
\hline+ & 15 & 8 & 23 & & & \\
\hline - & 17 & 5 & 22 & & & \\
\hline $\mathrm{N}$-cadherin & & & & 10.020 & 0.002 & 0.525 \\
\hline+ & 27 & 4 & 31 & & & \\
\hline- & 5 & 9 & 14 & & & \\
\hline Vimentin & & & & 3.380 & 0.066 & 0.274 \\
\hline+ & 17 & 3 & 20 & & & \\
\hline - & 15 & 10 & 25 & & & \\
\hline Snail1 & & & & 0.160 & 0.690 & 0.11 \\
\hline+ & 21 & 7 & 28 & & & \\
\hline - & 11 & 6 & 17 & & & \\
\hline Slug & & & & 11.948 & 0.001 & 0.515 \\
\hline+ & 23 & 2 & 25 & & & \\
\hline - & 9 & 11 & 20 & & & \\
\hline ZEB1 & & & & 1.625 & 0.202 & 0.19 \\
\hline+ & 19 & 5 & 24 & & & \\
\hline - & 13 & 8 & 21 & & & \\
\hline Twist & & & & 7.099 & 0.008 & 0.448 \\
\hline+ & 25 & 4 & 29 & & & \\
\hline- & 7 & 9 & 16 & & & \\
\hline Total & & 32 & 13 & 45 & & \\
\hline
\end{tabular}

MAOA, monoamine oxidase A; EMT, epithelial to mesenchymal transition; NSCLC, non-small cell lung cancer.

adjacent lung tissues (Figs. 1 and 2; Table II). Moreover, we further found that MAOA expression was correlated with clinical stages and lymph node metastases, while no relation could be established with sex, age, degree of differentiation, and histological types (Table IV). Taken together, our results suggest that MAOA may play a role in promoting the progression of NSCLC.

EMT, a key step in invasion and metastasis, plays a crucial role in the progression of cancers, including NSCLC (16-19). Wu et al (25) demonstrated that MAOA expression in prostate cancer suppressed epithelial phenotype and promoted mesenchymal transition by decreasing the expression of epithelial marker E-cadherin, while increasing the expressions of mesenchymal marker vimentin and transcription factor Twist, indicating its association with EMT in prostate cancer. In the present study, we showed that the increased MAOA expression in NSCLC tissues was negatively correlated with E-cadherin expression, but positively correlated with the expressions of N-cadherin, Slug, and Twist (Table III),
Table IV. Correlationship between the expression of MAOA and clinicopathologic characteristics.

\begin{tabular}{lrrrr}
\hline & & \multicolumn{2}{c}{ MAOA } & \\
Variables & $n$ & - & + & P-value \\
\hline Age (yeras) & & & & 0.691 \\
$<60$ & 13 & 3 & 10 & \\
$\geq 60$ & 17 & 6 & 11 & \\
Sex & & & & 1.000 \\
$\quad$ Male & 21 & 6 & 15 & \\
Female & 9 & 3 & 6 & \\
TNM stage & & & & 0.029 \\
I+II & 21 & 9 & 12 & \\
III & 9 & 0 & 9 & \\
Differentiation & & & & 0.687 \\
$\quad$ Poorly & 11 & 4 & 7 & \\
$\quad$ Moderatly & 19 & 5 & 14 & \\
Pathologic type & & & & 0.704 \\
$\quad$ Adenocarcinoma & 12 & 3 & 9 & \\
$\quad$ Squamous & 18 & 6 & 12 & \\
$\quad$ Lymph node metastasis & & & & 0.020 \\
$\quad$ Negative & 13 & 7 & 6 & \\
$\quad$ Positive & 17 & 2 & 15 & \\
$\quad$ Total & 30 & 9 & 21 & \\
\hline
\end{tabular}

MAOA, monoamine oxidase A.

suggesting that MAOA may mediate EMT, leading to the progression of NSCLC.

In conclusion, we demonstrated for the first time, to the best of our knowledge, that MAOA expression was significantly increased in NSCLC tissues, which was positively associated with EMT, late stages and lymph node metastases of the cancer, thus supporting the notion that MAOA may play a role in NSCLC progression by regulating the EMT process.

\section{Acknowledgements}

This study was supported by the grants from National Natural Science Foundation of China, 81372511) (to X.T.), Special Fund for Scientific and Technological Development (Basic and Applied Basic Research) of Guangdong Province (Natural Science Foundation of Guangdong Province), 2017A030313539 (to X.T.), "Sail plan" in Guangdong Province to cultivate high-level talents, 201635011 (to X.T.), Guangdong Provincial Department of Science and Technology (Research and Development of Industrial Technology in Guangdong Province), 2013B031100002 (to X.T.), and Zhanjiang Municipal Governmental Specific Financial Fund Allocated for Competitive Scientific and Technological Projects, 2012C0303-56 (to X.T.). We would like to thank Professor Han-Guo Jiang (Department of Pathology, Guangdong Medical University, China) and Dr. Ketao Jin (Shaoxin 
Hospital, Zhejiang, China) for their diagnoses performed on NSCLC tissues and their guidance regarding immunohistochemical staining methods.

\section{References}

1. Islami F, Torre LA and Jemal A: Global trends of lung cancer mortality and smoking prevalence. Transl Lung Cancer Res 4: 327-338, 2015.

2. Travis WD, Brambilla E, Burke AP, Marx A and Nicholson AG: The new IASLC/ATS/ERS international multidisciplinary lung adenocarcinoma classification. J Thoracic Oncol 6: 244-285, 2011.

3. Tang ER, Schreiner AM and Pua BB: Advances in lung adenocarcinoma classification: A summary of the new international multidisciplinary classification system (IASLC/ATS/ERS) J Thorac Dis 6 (Suppl 5): S489-S501, 2014.

4. Pikin OV, Ryabov AB, Trakhtenberg AK, Glushko VA, Kolbanov KI, Amiraliev AM, Barmin VV and Tukvadze ZG: Analysis of postoperative complications after pneumo-n-ectomy using thoracic morbidity and mortality (tmm) system in nsclc patients for a 5-year period. Khirurgiia (Mosk): 23-27, 2016 (In Russian). doi: 10.17116/hirurgia20161223-27.

5. Akthar AS, Ferguson MK, Koshy M, Vigneswaran WT and Malik R: Limitations of PET/CT in the detection of occult N1 metastasis in clinical stage $\mathrm{I}(\mathrm{T} 1-2 \mathrm{aN} 0)$ non-small cell lung cancer for staging prior to stereotactic body radiotherapy. Technol Cancer Res Treat 16: 15-21, 2017.

6. Renaud S, Falcoz PE, Olland A, Reeb J, Santelmo N and Massard G: Mediastinal downstaging after induction treatment is not a significant prognostic factor to select patients who would benefit from surgery: The clinical value of the lymph node ratio Interact Cardiovasc Thorac Surg 20: 222-227, 2015.

7. Yu S, Yan C, Yang X, He S, Liu J, Qin C, Huang C, Lu Y, Tian Z and Jia L: Pharmacoproteomic analysis reveals that metapristone (RU486 metabolite) intervenes E-cadherin and vimentin to realize cancer metastasis chemoprevention. Sci Rep 6: 22388, 2016.

8. Zhang X, Liang D, Fan J, Lian X, Zhao Y, Wang X, Chi ZH and Zhang P: Zinc attenuates tubulointerstitial fibrosis in diabetic nephropathy via inhibition of HIF through PI-3K signaling. Biol Trace Elem Res 173: 372-383, 2016

9. Liu S, Yang H, Chen Y, He B and Chen Q: Krüppel-like factor 4 enhances sensitivity of cisplatin to lung cancer cells and inhibits regulating epithelial-to-mesenchymal transition. Oncol Res 24: 81-87, 2016.

10. Jiang SB, He XJ, Xia YJ, Hu WJ, Luo JG, Zhang J and Tao HQ: MicroRNA-145-5p inhibits gastric cancer invasiveness through targeting N-cadherin and ZEB2 to suppress epithelial-mesenchymal transition. Onco Targets Ther 9: 2305-2315, 2016.

11. Zhou JP, Gao ZL, Zhou ML, He MY, Xu XH, Tao DT, Yang CC and Liu LK: Snail interacts with Id2 in the regulation of TNF- $\alpha$-induced cancer cell invasion and migration in OSCC. Am J Cancer Res 5: 1680-1691, 2015.

12. Li SP, Xu HX, Yu Y, He JD, Wang Z, Xu YJ, Wang CY, Zhang HM, Zhang RX, Zhang JJ, et al: LncRNA HULC enhances epithelial-mesenchymal transition to promote tumorigenesis and metastasis of hepatocellular carcinoma via the miR-200a-3p/ZEB1 signaling pathway. Oncotarget 7 : 42431-42446, 2016.

13. Ha JH, Ward JD, Radhakrishnan R, Jayaraman M, Song YS and Dhanasekaran DN: Lysophosphatidic acid stimulates epithelial to mesenchymal transition marker Slug/Snail2 in ovarian cancer cells via Gai2, Src, and HIF1 $\alpha$ signaling nexus. Oncotarget 7 : 37664-37679, 2016.

14. Zidar N, Boštjančič E, Jerala M, Kojc N, Drobne D, Štabuc B and Glavač D: Down-regulation of microRNAs of the miR-200 family and up-regulation of Snail and Slug in inflammatory bowel diseases - hallmark of epithelial-mesenchymal transition. J Cell Mol Med 20: 1813-1820, 2016.

15. Wang Y, Liu J, Ying X, Lin PC and Zhou BP: Twist-mediated epithelial-mesenchymal transition promotes breast tumor cell invasion via inhibition of hippo pathway. Sci Rep 6: 24606, 2016

16. Roy BC, Kohno T, Iwakawa R, Moriguchi T, Kiyono T, Morishita K, Sanchez-Cespedes M, Akiyama T and Yokota J: Involvement of LKB1 in epithelial-mesenchymal transition (EMT) of human lung cancer cells. Lung Cancer 70: 136-145, 2010 .
17. Che J, Yang Y, Xiao J, Zhao P, Yan B, Dong S and Cao B: Decreased expression of claudin-3 is associated with a poor prognosis and EMT in completely resected squamous cell lung carcinoma. Tumour Biol 36: 6559-6568, 2015.

18. Huang D, Duan H, Huang H, Tong X, Han Y, Ru G, Qu L, Shou C and Zhao Z: Cisplatin resistance in gastric cancer cells is associated with HER2 upregulation-induced epithelial-mesenchymal transition. Sci Rep 6: 20502, 2016

19. Sung WJ, Kim H and Park KK: The biological role of epithelial-mesenchymal transition in lung cancer (Review). Oncol Rep 36: 1199-1206, 2016.

20. Edgnülü TG, Özge A, Erdal N, Kuru O and Erdal ME: Association analysis of the functional MAOA gene promoter and MAOB gene intron 13 polymorphisms in tension type headache patients. Adv Clin Exp Med 23: 901-906, 2014.

21. Nikolac Perkovic M, Svob Strac D, Nedic Erjavec G, Uzun S, Podobnik J, Kozumplik O, Vlatkovic S and Pivac N: Monoamine oxidase and agitation in psychiatric patients. Prog Neuropsychopharmacol Biol Psychiatry 69: 131-146, 2016.

22. Liu Z, Huang L, Luo XJ, Wu L and Li M: MAOA Variants and genetic susceptibility to major psychiatric disorders. Mol Neurobiol 53: 4319-4327, 2016.

23. Voltas N, Aparicio E, Arija V and Canals J: Association study of monoamine oxidase-A gene promoter polymorphism (MAOA-uVNTR) with self-reported anxiety and other psychopathological symptoms in a community sample of early adolescents. J Anxiety Disord 31: 65-72, 2015.

24. Peehl DM, Coram M, Khine H, Reese S, Nolley R and Zhao H: The significance of monoamine oxidase-A expression in high grade prostate cancer. J Urol 180: 2206-2211, 2008.

25. Wu JB, Shao C, Li X, Li Q, Hu P, Shi C, Li Y, Chen YT, Yin F, Liao CP, et al: Monoamine oxidase A mediates prostate tumorigenesis and cancer metastasis. J Clin Invest 124: 2891-2908, 2014.

26. Wu JB, Yin L, Shi C, Li Q, Duan P, Huang JM, Liu C, Wang F, Lewis M, Wang Y, et al: MAOA-dependent activation of Shh-IL6-RANKL signaling network promotes prostate cancer metastasis by engaging tumor-stromal cell interactions. Cancer Cell 31: 368-382, 2017.

27. Stone L: Prostate cancer: Feel it in your bones: MAOA mediates metastasis. Nat Rev Urol 14: 326-327, 2017.

28. Lin YC, Chang YT, Campbell M, Lin TP, Pan CC, Lee HC, Shih JC and Chang PC: MAOA - a novel decision maker of apoptosis and autophagy in hormone refractory neuroendocrine prostate cancer cells. Sci Rep 7: 46338, 2017.

29. Zhao H, Flamand V and Peehl DM: Anti-oncogenic and pro-differentiation effects of clorgyline, a monoamine oxidase A inhibitor, on high grade prostate cancer cells. BMC Med Genomics 2: 55, 2009.

30. Wu JB, Lin TP, Gallagher JD, Kushal S, Chung LW, Zhau HE, Olenyuk BZ and Shih JC: Monoamine oxidase A inhibitor-near-infrared dye conjugate reduces prostate tumor growth. J Am Chem Soc 137: 2366-2374, 2015.

31. Li J, Yang XM, Wang YH, Feng MX, Liu XJ, Zhang YL, Huang S, Wu Z, Xue F, Qin WX, et al: Monoamine oxidase A suppresses hepatocellular carcinoma metastasis by inhibiting the adrenergic system and its transactivation of EGFR signaling. J Hepatol 60: 1225-1234, 2014

32. Huang L, Frampton G, Rao A, Zhang KS, Chen W, Lai JM, Yin XY, Walker K, Culbreath B, Leyva-Illades D, et al: Monoamine oxidase A expression is suppressed in human cholangiocarcinoma via coordinated epigenetic and IL-6-driven events. Lab Invest 92: 1451-1460, 2012.

33. Witalison EE, Cui X, Causey CP, Thompson PR and Hofseth LJ: Molecular targeting of protein arginine deiminases to suppress colitis and prevent colon cancer. Oncotarget 6: 36053-36062, 2015.

34. Wang J,Li J, Shen J, Wang C, Yang L and Zhang X: MicroRNA-182 downregulates metastasis suppressor 1 and contributes to metastasis of hepatocellular carcinoma. BMC Cancer 12: 227, 2012.

35. Różycka A, Słopień R, Słopień A, Dorszewska J, Seremak-Mrozikiewicz A, Lianeri M, Maciukiewicz M, Warenik-Szymankiewicz A, Grzelak T, Kurzawińska G, et al: The MAOA, COMT, MTHFR and ESR1 gene polymorphisms are associated with the risk of depression in menopausal women. Maturitas 84: 42-54, 2016. 